\title{
Research on Key Technology of Current Type Inverter Experimental Device
}

\author{
Ping Xin ${ }^{1, a}$, Dejun Liu ${ }^{1, b^{*}}$ and Hongtao $\mathrm{Mi}^{1, \mathrm{c}}$ \\ ${ }^{1}$ School of BeiHua University, Jilin 132021, China; \\ a359411897@qq.com, b14145277@qq.com, ${ }^{c} 17207527 @ q q . c o m$ \\ *The corresponding author
}

\begin{abstract}
The effect of Current type inverter is obvious in energy saving and consumption reduction, But the technology of domestic current type inverter is not mature enough, which is mostly imported from abroad. Imported inverter is expensive and high maintenance cost. Therefore, it is quite necessary to research and development current type inverter independently, resolves the technical difficulties in application and achieves its high performance. This has an important guiding value for the practical application of current mode inverter. The experimental device is aimed at the problem of harmonic pollution and dynamic performance in its technical difficulties, the global stability and non-singular point of the system is realized by using the system controller designed by the energy shaping control theory, which can improve the robustness of the system to parameter variations and external perturbations.
\end{abstract}

Keywords: Experimental technology research; Current type inverter; Energy shaping; Dual PWM; DSP

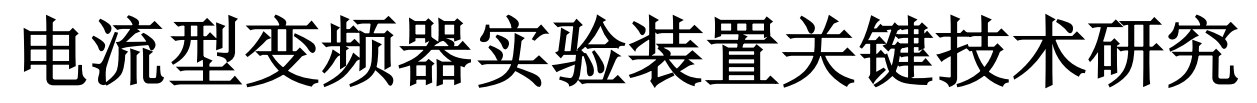

\author{
辛平 ${ }^{1}$ ，刘德君 ${ }^{1}$, 弭洪涛 ${ }^{1}$ \\ (1. 北华大学 电气信息工程学院, 吉林 吉林 132021)
}

摘要: 电流型变频器在节能降耗方面效果明显, 但目前国产电流型变频器技术不够成熟, 大多从国外进口, 进口变频器 价格昂贵, 维修费用高。因此, 自主研发电流型变频器实验装置, 解决应用中的技术难点, 实现其高性能很有必要, 对电流 型变频器的实际应用具有重要的指导价值。本实验装置针对其技术难点中的谐波污染和动态性能问题, 利用能量成型控制理 论设计的系统控制器实现系统的全局稳定性，无奇异点，提高系统对参数变化及外来摄动的鲁棒性。

关键词: 实验技术研究; 电流型变频器; 能量成型; 双 PWM；DSP

中图分类号: TM921.51 文献标志码: A

引言

近些年来, 电流源型双 PWM 变频器, 因具有四象限运行能力、可实现可靠的短路保护, 运行效率高等 特点, 使之在在大功率场合具有显著的优势, 相关控制技术成为国内外的研究热点。变频器的发展道路还 是十分广阔的, 同时还有好多的问题等待我们去解决 [1], 这就需要科研技术人员们做更多更深入的研究, 只有这样才能使我们的工业技术和科技水平达到先进国家的水平。随着芯片 DSP 处理运行速度的大大提升, 在电流源型变频器控制系统中研究一些新型的控制算法 [2, 3], 并进行实验验证, 加快其完善和应用的步 伐。

随着电力电子技术和超导磁储能技术的发展, 电流源型变频器凭着自身的一系列优点已经在交流传动 领域获得了认可 $[4,5]$, 且具有光明的应用前景, 加大加快对电流源型变频器关键技术的研究与攻关, 使 我国的电流源型变频器技术达到世界先进水平, 使该产品具有更好更高的工作性能, 这将十分具有科研价 
值和实际意义。

\section{1 基于 DSP 的电流型变频器实验原理}

Ti DSP2812 是一款功能强大，专门用于运动控制开发的芯片。其片内有可以用来专门生成 PWM 波 [10] 的事件管理单元 EVA、EVB, 配套的 12 位 16 通道的 AD 数据采集, 丰富的 CAN、SCI 等外设接口, 为电流型 变频器的开发提供了极大地便利。基于 DSP 的电流型变频器调速系统框图如图 1 所示。

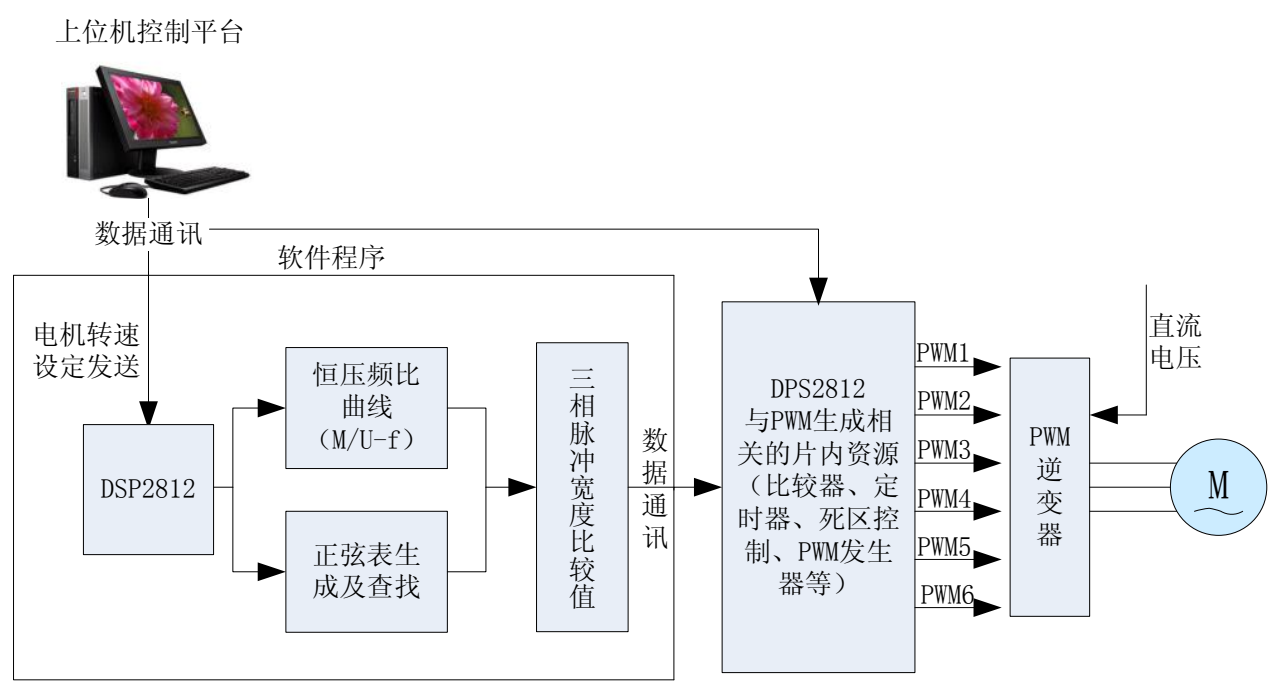

图 1 基于 DSP 的电流型变频器调速系统

系统上位机发送转速设定值及其他运行参数到 DSP 片内, 采用矢量控制对交流电机数学模型进行解耦, 使用 SVPWM 调制算法形成六路触发脉冲对开关管通断进行控制[6]。以 DSP TMS320F2812 为核心，搭建实 验平台, 并利用 CCS 软件进行程序开发, 完成对不同控制算法的编写, 验证理论分析和仿真结果的正确性, 并不断完善所提出的理论与技术方案。

\section{2 实验技术与方法}

由于电流源型变频器是一个多变量、非线性、强耦合的复杂系统 [7], 要想直接获得预期的控制效果 比较困难, 因此, 该项目必须在深入研究理论和不断推导论证的基础上, 经过反复设计与实验, 才能逐步 实现系统的高性能控制效果, 完成最终研究目标。

\section{1 新型 SVPWM 调制算法的理论研究}

开关时间预估模型以精确采样点位置为思想 [8]、以减少计算误差为目标最终实现降低谐波含量。经 过申请人充分的分析与初步研究认为: 对扇区内每一个工作矢量作为计算对象, 根据调制序列的安排分别 设定采样点位置, 相比于传统的扇区所有工作矢量使用相同采样点位置来说, 开关时间计算结果误差将大 大降低，进而大幅降低谐波含量。图 2 所示为开关时间预估模型原理图。 


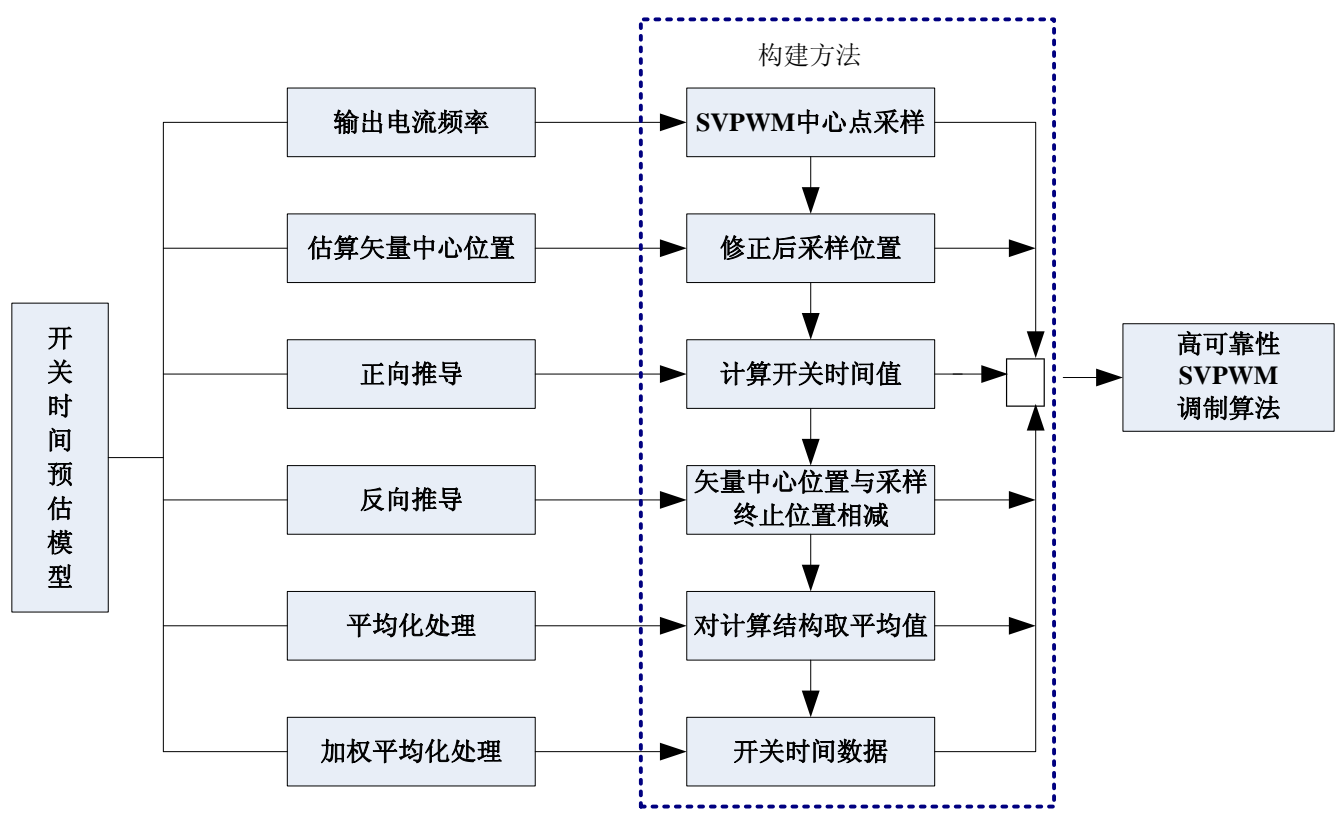

图 2 开关时间预估模型原理示意图

针对传统 SVPWM 存在的谐波影响, 精确计算采样点位置, 构建一种开关时间预估模型。基于该模型, 改进传统 SVPWM 调制算法, 实现电流型变频器低谐波含量与高工作效率。

分析传统的 SVPWM 调制算法, 研究适合于电流源型变频器的调制序列, 运用二重傅里叶积分理论求解 谐波函数表达式 $[9,10]$, 总结传统 SVPWM 方法的谐波特性, 寻找存在的缺点与不足, 探讨降低谐波含量 的途径与突破口。

\section{2 能量成型控制策略研究}

采用基于端口受控耗散哈密顿 (PCHD) 模型的互联和阻尼注入配置的无源控制 (IDA-PBC) 方法 [11]，通 过能量成型原理和参数匹配方案设计 IDA-PBC 控制器, 实现电流源型整流器在交流侧运行于单位功率因 数、输出侧直流电流快速达到期望值并保持基本稳定。图 3 为能量成型控制策略示意图。

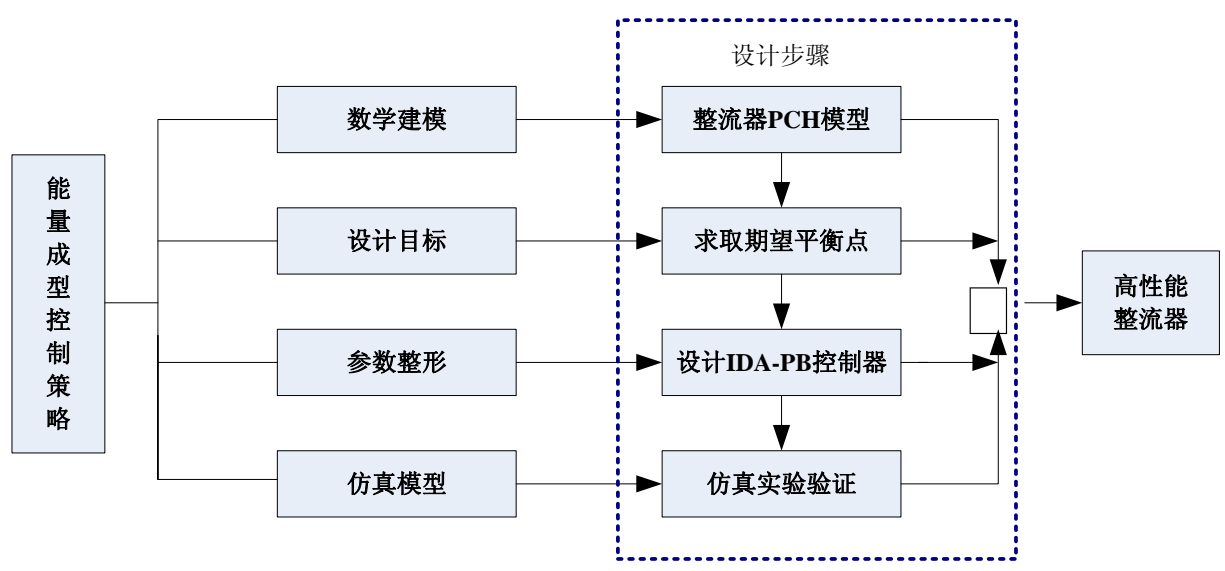

图 3 能量成型控制方案示意图

分析三相电流源型整流器的工作原理, 建立其动态数学模型, 基于 Hami1ton 方程探讨和建立电流源 型整流器的闭环状态 PCH 系统数学模型 [12]。以提高工作效率和功率因数为目标, 从能量损失最小化和 
最小磁场能量角度提高功率因数。利用系统的能量函数作为 Lyapunov 函数, 保证系统的稳定性。确定系 统期望稳定的平衡点（期望轨迹），设计合理的控制律，确保系统的动态响应性能并能快速收敛到期望平 衡点。

\section{3 实验装置应用转化的前景预测}

\section{1 减少能源浪费、提高装置转换效率的工业生产需求}

电流型变频器采用变频调速技术可以使大中型变工况电机获得优良调速性能, 这大大不仅减少采用直 接拖动电机所带来的资源和能源浪费, 而且达到了很好的节能效果。然而, 当电流源型变频器应用于大容 量交流调速场合时，其本身转换效率的高低对造成能源和资源的浪费也不可忽视。另外，较低的转换效率 除了对能源造成浪费外, 还会影响系统的性能, 使电力电子开关器件因过热而损坏。因此, 提高电流源型 变频器装置转换效率，减小电力电子开关器件损耗和谐波损耗对系统很必要 [13]。

\section{2 高性能变频调速系统的要求}

变频调速作为一种具有效率高、调速平稳、调速范围宽、无极变速等优点的调速技术，在大功率中高 压交流传动场合，如扎钢、水泥、煤炭、造纸、船舶及铁路等工业领域中，该技术应用十分泛。电流源型 变频器作为中高压大功率交流电动机调速的主要驱动装置, 其显著特点是容易实现回馈制动和四象限运 行, 尤其在大容量可逆轧机、卷扬机等工业场合的应用, 通过控制其内部功率开关器件的开通与关断, 将 电压幅值和频率固定的工频电变换成幅值和频率可调的交流电, 以至于实现对轧机的精确调速控制, 从而 更好地改善生产工艺条件, 提高生产效率和产品质量。因此, 电流源型变频器的性能的好坏直接决定着交 流电动机调速的效果。

\section{4 结束语}

实验室是是科学研究的基地，科技发展的源泉，对科技发展具有重要的价值。新型实验装置的研发不 仅代表科技的进步，同时对工业的发展和国家的发展发挥着重要的作用。在实验装置的开发过程中融合创 新思想和方法，实现关键技术创新，取得具有自主知识产权的研究成果，能更加体现出科技的价值。

\section{5 致谢}

这项研究得到吉林省教育厅科技技术研究项目（201656）；吉林省教育厅基金项目（201632）；教育 部高校学校电气类专业教学指导委员会 (DQJZW2016002) 的支持; 北华大学教育课题 (BHDQ31) 的支持。

\section{Acknowledgement}

This research was financially supported by Jilin Provincial Department of Education Science and technology research project(201656); The Education Department Project of Jilin Province(201632); The Education Department of the Ministry of education higher education professional teaching steer(DQJZW2016002); Education and teaching research topics of Beihua University (BHDQ31).

\section{参考文献:}

[1］何钦, 张志山．变频器谐波的危害及防治 [J]．电机与控制应用，2012，39(11)：54-57.

[2] 吴文宣，吴丹岳，林炎. 电流型变频器的间谐波分析及应用模型构建 $[\mathrm{J}]$. 电工技术学报. 2010，25 (2) : 163-176.

［3］李和明，朱晓荣．三相电流型 PWM 控制器的控制方法发展综述 [J]。华东电力，2013，41 (2)：406-411.

[4] 舟岩，宋蕙慧，曲延滨．光伏并网发电系统的能量成型控制策略研究 [J]．电测与仪表，2014，51 (2)：36-39.

[5] 石健将, 王文杰, 龙江涛. 三相四线 PWM 整流器的一种新型零静差控制策略研究 [J]. 电工技术学报, 2013. 6, 
$28(6): 114-119$.

[6] 王克柔, 吴燕仙, 李玉玲. 一种电流型三相逆变器 PWM 调制的数学实现 $[J]$. 电力电子技术, 2011, Vol. 45, No. 4:76-78.

[7] K. Wei, Z. Lixia, W. Yansong. Study on output characteristic of bi-direction current source converters [J]. IET Power Electronics, 2011, 5(7) : 929-934.

[8] Fernando Castaños, Romeo Ortega. Asymptotic stabilization via control by interconnec- tion of port-Hamiltonian systems[J]. Automatica, 2009, 45(7): 1611-1618.

[9] Luiz A C, Maged F N. Space-Vector-Modulated Hybrid Bidirectional Current Source Converter[J]. IEEE Transactions on Power Electronics, 2010, 25(4): 1055-1067.

[10] Navid R, Xinbo Ruan. A Natural Soft-Commutation PWM Scheme for Current Source Converter and Its Logic Implementation [J]. IEEE Transactions on Industrial Electronics, 2011, 58 (7) : 2772-2779.

[11] Popat, Miteshkumar, Navid R. Fault ride-through capability of cascaded current-source converter-based offshore wind farm [J]. IEEE Transactions on Sustainable Energy, 2013, 4(2) :314-323.

[12] David Xu. Control Strategy With a Generalized DC Current Balancing Method for Multimodule Current-Source Converter [J]. IEEE Transactions on Power Electronics, 2014, 29(1) : 366-373.

[13] DAISUKE GUNJI, TAKEHIRO IMURA. Fundamental Research on Control Method for Power Conversion Circuit of Wireless In-Wheel Motor Using Magnetic Resonance Coupling [J]. Elect Eng Jpn, 2016, 30(6) :1954-1967.

\section{References}

[1] He Qin, Zhang Zhi-shan. Harm and prevention and control of the application of [J]. motor, inverter harmonic, Vol. 39(2012), No.11, p.54-57 (in Chinese)

[2] Wu Wen-xuan, Wu Dan-yue, Lin Yan. Analysis and application model of inter harmonic analysis of current mode inverter [J]. Transactions of China Electrotechnical Society, Vol. 25(2010), No.2, p.163-166 (in Chinese)

[3] Qing Tai-quan:Common DC regulated power supply circuit used in 200 cases (China power press,China 2013), p.10-22. (in Chinese)

[4] Ran Yan, Song Hui-hui, Qu Yan-bin. Photovoltaic power generation system, energy shaping control strategy of $[\mathrm{J}]$. electrical measurement and instrumentation, Vol. 51(2014), No.2, p.36-39 (in Chinese)

[5] Shi Jian-jiang, Wang Wen-jie, Long Jiang-tao. A novel zero static error control strategy for three-phase four wire PWM rectifier [J]. proceedings of the Chinese society of electrical engineering, Longjiang, Vol. 28(2013), No.10, p.114-119 (in Chinese)

[6] Wang Ke-rou, Wu Yan-xian, Li Yu-ling. A mathematical realization of PWM modulation for current source three-phase inverter [J]. power electronics technology, Vol. 45(2011), No.4, p.76-78 (in Chinese)

[7] K. Wei, Z. Lixia, W. Yansong. Study on output characteristic of bi-direction current source converters[J]. IET Power Electronics, Vol. 5(2011), No.7, p.929-934

[8] Fernando Castaños, Romeo Ortega. Asymptotic stabilization via control by interconnec- tion of port-Hamiltonian systems[J]. Automatica, Vol. 45(2012), No.7, p.1611-1618

[9] Luiz A C, Maged F N. Space-Vector-Modulated Hybrid Bidirectional Current Source Converter[J]. IEEE Transactions on Power Electronics, Vol. 25(2011), No.4, p.1055-1058 
[10] Navid R, Xinbo Ruan. A Natural Soft-Commutation PWM Scheme for Current Source Converter and Its Logic Implementation[J]. IEEE Transactions on Industrial Electronics, Vol. 58(2011), No.7, p.2772-2779

[11] Popat, Miteshkumar, Navid R. Fault ride-through capability of cascaded current-source converter-based offshore wind farm[J]. IEEE Transactions on Sustainable Energy, Vol. 4(2013), No.2, p.314-317

[12]David Xu. Control Strategy With a Generalized DC Current Balancing Method for Multimodule Current-Source Converter[J]. IEEE Transactions on Power Electronics, Vol. 29(2014), No.1, p.366-373

[13]DAISUKE GUNJI,TAKEHIRO IMURA.Fundamental Research on Control Method for Power Conversion Circuit of Wireless In-Wheel Motor Using Magnetic Resonance Coupling[J]. Elect Eng Jpn, Vol. 30(2016), No.6, p.1954-1967 